Infantile onset spinocerebellar ataxia. Identification of a novel Twinkle mutation is reported in a family with infantile onset spinocerebellar ataxia in two individuals manifesting ataxia, peripheral sensory neuropathy, athetosis, seizures, deafness, and ophthalmoplegia (Dundar H, et al. Pediatr Neurol 2012 Mar;46(3):172-7).

\title{
PATHOPHYSIOLOGY OF PRIMARY AND SECONDARY DYSTONIAS
}

Investigators at the Institute of Neurology, University College London, UK, and centers in Slovenia and Spain compared electrophysiological features of primary and secondary dystonia, using transcranial magnetic stimulation of motor cortex and eye blink conditioning. Eleven patients with hemidystonia secondary to basal ganglia or thalamic lesions were tested over both hemispheres, corresponding to the affected and nonaffected sides, and compared with 10 patients with primary segmental dystonia with arm involvement and 10 healthy controls. All subjects were tested as adults. The average age at onset of secondary dystonia was 13.6 years (range $1-55 ; 5$ were $1-2$ years). The causes of secondary dystonia were perinatal $\mathrm{HII}$ in 5, ischemic stroke in 5, and encephalitis in 1 .

No differences in motor thresholds were detected between patients with secondary and primary dystonia or controls. In secondary dystonia, short interval intracortical inhibition was reduced on the affected side, but normal on the non-affected side; cortical plasticity and eye blink classical conditioning were normal. In contrast, patients with primary dystonia showed increased cortical plasticity and reduced eye blink classical conditioning. Dystonia is a motor symptom that reflects different pathophysiological mechanisms. (Kojovic M, Parees I, Kassavetis P, et al. Secondary and primary dystonia: pathophysiological differences. Brain 2013 Jul;136(Pt 7):2038-49). (Response: Dr Maja Kojovic. E-mail: maja.kojovic.09@ucl.ac.uk).

COMMENT. Adams and Victor's Principles of Neurology $\left(9^{\text {th }}\right.$ ed. New York: McGraw Hill Medical; 2009) lists 4 main groups of diseases characterized by dystonia: 1) Hereditary and Degenerative dystonias (dystonia musculorum deformans, Huntington chorea, Juvenile dystonia - Parkinson syndrome [L-dopa responsive]), 2) Drug-induced (phenothiazine, haloperidol), 3) Symptomatic (secondary) (Wilson disease, double athetosis cerebral palsy due to cerebral hypoxia, kernicterus), and 4) Idiopathic focal dystonias (spasmodic torticollis, blepharospasm, hemifacial spasm, oromandibular, spasmodic dysphonia, writer's cramp).

\section{BIOTIN-RESPONSIVE OPHTHALMOPLEGIA / DYSTONIA}

A 10-year-old girl with a 4-month history of abnormal gait and dysarthria had bilateral external ophthalmoplegia, dystonia, and altered mental status. MRI showed a characteristic "bat-wing" appearance and increased signal involving the medial nucleus of the thalamus, basal ganglia and cerebellum, suggesting biotin-responsive basal ganglia disease. Immediate improvement followed biotin and thiamine therapy. Repeat MRI showed resolution of vasogenic edema but residual atrophy and gliosis in the basal ganglia. The disease is autosomal recessive with SLC19A3 gene mutation, related to 